\title{
LA POLÍTICA EXTERIOR DE ESPAÑA HACIA EL MEDITERRÁNEO EN LA ÚLTIMA DÉCADA. UNA EVALUACIÓN
}

\author{
Antonio Marquina ${ }^{1}$ \\ Director de UNISCI
}

\begin{abstract}
Resumen:
La política de España hacia el Mediterráneo ha tenido desde mediados de los años noventa un componente bilateral y un fuerte componente multilateral. El artículo explica los cambios y transformaciones producidos en el espacio mediterráneo en la última década y explica las políticas puestas en práctica y los errores y carencias detectables. El artículo señala la necesidad de repensar y planificar de forma más rigurosa las políticas a desarrollar por España en los próximos años.
\end{abstract}

Palabras clave: Política exterior de España, Mediterráeo.

Title in English: "Spain's Foreign Policy towards the Mediterranean. An Assessment".

\begin{abstract}
:
Spain's policy towards the Mediterranean has maintained since the mid-nineties a bilateral component and a strong multilateral component. The article explains the changes and transformations that took place in the Mediterranean in the last decade and explains the policies implemented, including an assessment of detectable errors and shortcomings. The article points out the need to rethink and plan more rigorously the policies to be implemented by Spain in the next coming years.
\end{abstract}

Keywords: Spain's Foreign Policy, Mediterranean.

Copyright $@$ C UNISCI, 2011.

Las opiniones expresadas en estos artículos son propias de sus autores, y no reflejan necesariamente la opinión de UNISCI. The views expressed in these articles are those of the authors, and do not necessarily reflect the views of UNISCI.

\footnotetext{
${ }^{1}$ Antonio Marquina es Director de UNISCI y catedrático de Seguridad y Cooperación en Relaciones Internacionales en la Universidad Complutense de Madrid. Sus principales áreas de investigación son: seguridad en Europa, el Mediterránea, Asia-Pacífico y control de armamentos. E-mail: marioant@cps.ucm.es. 


\section{Introducción}

La política exterior española hacia el Mediterráneo ha tenido un componente bilateral y multilateral desde mediados de los años noventa. El Mediterráneo es una zona de interés preferente en la política española y esto explica la complejidad de relaciones y compromisos que España tiene en esta región y lo complicado de llevar a cabo políticas coherentes, bien pensadas y planificadas, asunto donde España muestra serias y, en algún caso como veremos, muy serias carencias, que han acabado afectando a los intereses nacionales. La política exterior como la de defensa exige excelentes expertos de reconocida valía intelectual, con una preparación auténticamente multidisciplinar en los centros de planificación, centros, por otra parte, escasamente dotados en España. Y exigen también una dirección muy exigente, con menos improvisación, más conocimiento de los temas y dosieres del Ministerio o Ministerios, y más tiempo para la reflexión. Algunas de las políticas y realizaciones de la última década, como veremos, permiten cuestionar no sólo su puesta en práctica, sino la planificación desarrollada y los apoyos y alianzas buscados o mantenidos.

\section{De la Conferencia de Barcelona a la Unión por el Mediterráneo}

Durante la presidencia española de la UE en el segundo semestre de 1995 tuvo lugar la Conferencia de Barcelona sobre el Mediterráneo, lanzándose un proceso para afrontar los nuevos desafíos políticos, económicos y sociales en el Mediterráneo. Asimismo España contribuyó de forma muy significada al lanzamiento del diálogo mediterráneo de la OTAN en 1994. Posteriormente, una vez finalizada la guerra civil argelina, y levantadas las sanciones a Libia por los atentados terroristas de un avión de la PANAM y otro de la UTA, el diálogo 5+5 reanudó su andadura a partir de 2001. Asimismo se desarrollaron otras iniciativas como el Foro Mediterráneo.

Una evaluación desapasionada de estos procesos y aproximaciones nos presenta un panorama poco convincente en cuanto a realizaciones. El proceso de Barcelona entra rápidamente en crisis en la cumbre de Malta de 1996, al irrumpir el conflicto entre israelíes y palestinos en la conferencia y se intenta revitalizar bajo presidencia británica, en la reunión ministerial de Palermo, el 3 y 4 de junio de 1998. En el discurso-declaración de Robin Cook, que quedó consensuado, se dejó bien sentado que el proceso de Barcelona y el proceso de paz de Oriente Medio tenían que ser considerados como complementarios, añadiendo un largo párrafo sobre la preocupación y el compromiso de los participantes en una paz justa y duradera, incluyendo el principio de tierra por paz. Luego, se intentaron abrir nuevas vías. Por primera vez se introdujo el concepto de medidas de asociación, que nuestro grupo de investigación UNISCI había propugnado y promovido, pero dándole un significado poco apropiado y poco ambicioso, convirtiéndolo luego los altos funcionarios en un concepto de vuelo raso, desnaturalizado y poco operativo. Y se llega a la cumbre ministerial de Stuttgart en abril de 1999 con la aprobación de las guías para la Carta de paz y estabilidad, Carta que la posterior presidencia francesa no puede conseguir consensuar en Marsella al iniciarse la segunda intifada. A partir de este momento el proceso de Barcelona queda ya muy tocado en cuanto a sus posibilidades, irrumpiendo en el proceso de forma absolutamente condicionante, el conflicto palestino-israelí y árabe-israelí.

Posteriormente tendrán lugar los atentados terroristas del 11 de septiembre de 2001, induciendo la adopción de nuevas políticas y nuevas aproximaciones al mundo musulmán a nivel global. 
España aprovechará la presidencia de la UE en 2002 para relanzar el proceso de Barcelona con la declaración de Valencia, el plan de actuación, la creación de una Asamblea Parlamentaria Euro-mediterránea y de una Fundación Euro-mediterránea para el Diálogo entre las Civilizaciones, para al año siguiente entrar en un proceso bastante irreconocible para los Estados árabes, como consecuencia de la guerra y ocupación de Iraq por Estados Unidos y su coalición. El cerrado alineamiento del gobierno de Aznar con la política de la Administración Bush en el asunto de Irak, aparte de chocar con las políticas y posiciones anteriormente mantenidas por España en las relaciones con los gobiernos de Saddam Hussein, significaba desmarcarse también de la opinión pública española que abrumadoramente estaba contra la guerra y entrar en colisión con el eje franco-alemán en el caso de la Unión Europea. Era un cambio casi copernicano en la política exterior.

A partir de esta guerra y con el lanzamiento de la política de vecindad europea en 2004, el proceso de Barcelona quedó ya muy tocado. En el capítulo político se había privilegiado desde el principio la economía sobre la política y hubiese sido fundamental cambiar esta orientación. En el mismo capítulo, en el campo de la seguridad, se había intentado proyectar la agenda europea en un espacio de seguridad muy mal diseñado para el tratamiento de las armas de destrucción masiva y se habían adoptado principios y conceptos que no podían ser operativos. Hay que resaltar un notable inmovilismo en el tratamiento de las cuestiones de seguridad, de forma especial, pero no única, en la seguridad militar y las armas de destrucción masiva. En estos asuntos no hubo ningún intento de hacer un replanteamiento que la nueva situación en el Mediterráneo Oriental hacía perentorio ${ }^{2}$.

De esta forma, al cumplirse el décimo aniversario de la firma de la Declaración de Barcelona, el nuevo gobierno español trató de relanzar el proceso de Barcelona.

Un balance desapasionado incluía los siguientes elementos:

- Escasos avances en la transformación de los regímenes autoritarios de los estados del sur del Mediterráneo.

- Políticas contradictorias de apoyo a la democratización por parte de la Unión Europea.

- Liberalización comercial favorable a los Estados de la Unión Europea.

- Muy limitados avances en los intercambios sur-sur.

- Incremento de la desigualdad económica entre el norte y el sur del Mediterráneo.

- Fracaso inicial de la asignación de fondos MEDA.

- Fracaso en la modernización de la infraestructura económica de los estados del sur del Mediterráneo como motor para la atracción de la inversión privada.

- Fracaso en la regulación de los flujos migratorios.

- Muy escasa efectividad del diálogo cultural al ser selectivo y esencialmente unidireccional, sin un compromiso e interés efectivo de los estados del Sur del Mediterráneo, y centrado en productos culturales.

- Diálogo cultural orientado a las minorías intelectuales más cercanas a los estados occidentales, sin que estas minorías tengan tampoco en múltiples casos un compromiso serio con estos diálogos, contentándose en la mayoría de los casos a figurar como socios en actividades en las que de hecho participan como un elemento casi de adorno para viajes, reuniones y discusiones.

\footnotetext{
2 Véase a este respecto Marquina, Antonio: "La asociación de seguridad de la Conferencia Euro-Mediterránea diez años después: la anábasis", UNISCI Discussion Papers, nº.9 (octubre de 2005), en http://www.ucm.es/info/unisci/revistas/UNISCI9Marquina.pdf.
} 
El resultado de la cumbre de jefes de Estado y de Gobierno, en el décimo aniversario de la conferencia de Barcelona, el 27 y 28 de noviembre de 2005 fue algo decepcionante y el encuentro bastante caótico. No supuso una auténtica renovación. Los Estados árabes enviaron representantes de segundo nivel. Y, aunque la cumbre se realizó bajo presidencia británica, el equipo del Ministerio de Asuntos Exteriores no hizo un análisis adecuado de las posibilidades y avances que eran posibles realizar en aquel momento, tras la ocupación de Irak y el desarrollo de la insurgencia en este país que empeoraron la solución del conflicto árabe-israelí y palestino-israelí . El hecho de que al final de la cumbre se tuviera que recurrir a una declaración de la presidencia, recogiendo párrafos enteros de la declaración de Barcelona de 1995, fue revelador de la situación en que se encontraba el proceso. El énfasis inicial en esta declaración en el proceso de paz árabe-israelí y palestino-israelí no dejaba de ser también revelador de la situación. Hay que hacer notar, no obstante, la aprobación de un programa de trabajo a cinco años y un código de conducta de lucha contra el terrorismo, que si bien dejaba en el aire diversos elementos, significaba un paso adelante ${ }^{3}$.

Por parte española, el nuevo equipo del Ministerio de Asuntos Exteriores mantenía una filosofía consistente en avanzar en cooperaciones concretas, siguiendo la política de pequeños pasos, en espera de llegar un día a conceptualizaciones de conjunto. La ceguera era colosal, dado que, independientemente de la falta de resultados de cierta entidad, los cambios introducidos en el Mediterráneo con la creciente presencia de actores no europeos y la guerra de Irak hacían perentorio un cambio de filosofía y planteamientos que permitieran desarrollar las cooperaciones o políticas más prioritarias en un conjunto bien estructurado y con un objetivo bien delimitado.

De esta forma desde 2005 a 2008, cuando Francia lanza la iniciativa "Unión para el Mediterráneo" asistiremos a un proceso inercial, tratando de evitar que la política europea de vecindad y que la nueva asociación estratégica para el Mediterráneo y Oriente Medio desvirtuase esta iniciativa y, posteriormente, a un intento de acomodarse a la nueva filosofía que intentaba introducir Francia, intentando mantener el proceso de Barcelona y la impronta europeísta que había permitido a España jugar un papel relevante en el proceso. Esto puede ejemplarizarse en las manifestaciones del Ministro de Asuntos Exteriores en Malta en 2007, cuando se estaba fraguando la iniciativa de Sarkozy. Según la reseña oficial, el ministro de Asuntos Exteriores, en su discurso académico, tras recibir el doctorado honoris causa por la Universidad de La Valetta, abogó por que los países mediterráneos se movilizaran en la búsqueda de la paz en el conflicto de Oriente Medio. También apostó por relanzar la agenda mediterránea y avanzar hacia una "Comunidad Euro-mediterránea" basada en "valores compartidos". En esta línea, el ministro español pidió la puesta en marcha de un Consejo Euro-mediterráneo en el que los jefes de Estado y de Gobierno tomaran decisiones estratégicas y políticas. Una comisión se encargaría principalmente de las responsabilidades de orden socioeconómico, como la promoción de una zona de libre cambio y libre circulación para el año 2010 y consolidaría y expandiría también el espacio de libertad, seguridad y justicia creado en la Cumbre de Barcelona de 2005. Una Asamblea Parlamentaria, el "ágora euro-mediterránea", sería el hogar común de la democracia y los derechos y libertades de los ciudadanos euro-mediterráneos.

\footnotetext{
3 Véase, "10th Anniversary Euro-Mediterranean Summit", Chairmain's Statement, Barcelona (27 and 28 November 2005), en http://www.consilium.europa.eu/policies/foreign-policy/third-countries-and-regions/the-euand-the-mediterranean-region.aspx?lang=en.
} 
Estos planteamientos de "valores compartidos" y "Comunidad Euro-mediterránea" iban más allá de lo hasta entonces propugnado por el proceso de Barcelona: una asociación, pero, como en tantos discursos y manifestaciones españolas, no tenía ningún planteamiento de fondo detrás que diera credibilidad a estas afirmaciones. Más interesante era la aceptación de la necesidad de institucionalización del proceso de Barcelona, manteniendo la primacía de la Unión Europea sobre otras políticas nacionales. Pero el "nuevo espacio geopolítico capaz de responder con mayor eficacia a los desafíos del siglo XXI" que se pretendía desarrollar exigía una mayor homologación e interacción de los Estados no europeos para dar eficacia al proceso, independientemente de que el espacio mediterráneo en términos económicos ya era un espacio de mayor competición económica de actores no europeos.

$\mathrm{Al}$ año siguiente, la aprobación de la iniciativa de Sarkozy "Unión por el Mediterráneo" significaba ya un cambio institucional importante y un cambio de filosofía en una dirección más correcta, centrada en proyectos comunes, que devaluaba la relevancia que España había querido mantener en el proceso y la política de pequeños pasos que, estaba suficientemente claro, no producían avances apreciables, faltando un diseño de a dónde se quería ir, cómo afrontar de forma efectiva los desafíos políticos, económicos, sociales y también geopolíticos en el Mediterráneo que se encontraba en notable transformación, y, de forma especial, cómo avanzar en la solución del conflicto palestino-israelí y árabe israelí, donde tanto España como, de forma especial, la Unión Europea seguían manteniendo una política de gesticulación, incapaces de encauzar mínimamente estos conflictos y utilizar las poderosas palancas económicas de que la Unión Europea dispone. La Unión Europea, dada su división, se había convertido en un convidado de piedra. Había que romper con esta situación y salir de la gesticulación en el Cuarteto.

La reorientación de la iniciativa mediterránea realizada por Francia significaba el reconocimiento de fallos de cierta envergadura. Dejaba ya de lado un engranaje como EuroMesco que se había mostrado poco operativo y que no había llegado a ser un laboratorio de ideas adecuado, siendo su contribución en el relanzamiento del primer capítulo poco imaginativa y, sobre todo, poco operativa. En el campo económico la brecha entre el norte y el sur del Mediterráneo había crecido sustancialmente; nuevos actores no europeos eran cada vez más activos en la zona y era cuestión de afrontar esta cuestión crucial de forma diferente. Y en el campo social y cultural el diálogo se había centrado en los productos culturales, teniendo como interlocutores las élites occidentalizadas y los grupos más laicos a los que artificialmente se pretendió dar un peso que no tenían en las sociedades árabes. La cuestión migratoria se había acabado de encauzar mínimamente, pero de forma reactiva.

Teniendo en cuenta las experiencias anteriores hubiera sido necesario una política más imaginativa española o una crítica española más severa a la iniciativa "Unión por el Mediterráneo". Este no fue el caso. El tema del desarrollo político no aparecía en la iniciativa e incluso se mantenía literalmente el primer capítulo del texto de la conferencia de Barcelona. Era algo aberrante ${ }^{4}$. Los riesgos a la seguridad inducidos por la creciente apatía de la gente, en especial de jóvenes diplomados y de la baja clase media, la pérdida de control político y la búsqueda de alternativas no se tomaron en consideración. A esto vino a añadirse la crisis

\footnotetext{
4 Véase Marquina, Antonio: "La Declaración Conjunta de la Cumbre de París para el Mediterráneo. Un Documento Claramente Mejorable en Asuntos de Seguridad", UNISCI Discussion Papers, no 19 (enero 2009), en

http://www.ucm.es/info/unisci/revistas/UNISCI\%20DP\%2019\%20-

\%20MARQUINA\%20MEDITERRANEO.pdf.
} 
económica, la falta de avances en el proceso de paz entre árabes e israelíes y el arreglo institucional.

España consiguió la secretaría de la Unión, pero el sistema institucional no resultó suficientemente operativo para hacer frente a la nueva situación, teniendo en cuenta la carencia de medios adecuados, los planteamientos árabes y la presencia institucional de Israel. Tampoco Egipto fue suficientemente permeable a los intereses y planteamientos españoles.

Con las revueltas árabes el proceso ha quedado congelado y habrá que pensar en iniciativas mejor pensadas y más adecuadas al nuevo contexto mediterráneo que se abre, evitando dilapidar esfuerzos y dinero del contribuyente.

En el diálogo mediterráneo de la OTAN, España ha tenido un protagonismo de cierta relevancia, primero en su lanzamiento en 1994, luego en su impulsión, intentando darle un mayor contenido, tanto en le cumbre de Madrid de 1997, como en la cumbre de Washington de 1999, como en la cumbre de Praga de 2002. Como en el caso de la Conferencia de Barcelona, a pesar de los esfuerzos y los programas anuales de actividades, crecientemente abultados, el problema ha consistido en encontrar un encuadre y planteamientos adaptados a los cambios internacionales producidos. Curiosamente la ministro de Defensa, Carmen Chacón, ha reconocido recientemente que los Estados del diálogo mediterráneo no saben qué pedir y los Estados de la OTAN no saben qué ofrecer. Esta situación es sencillamente el reconocimiento de un mal planteamiento.

España también ha apoyado el diálogo $5+5$ que ha desarrollado numerosas actividades de forma especial a partir de la Cumbre de Túnez de 2003 donde se centraron los asuntos de cooperación:

- Estabilidad y seguridad en el mediterráneo Occidental.

- Cooperación económica e integración magrebí.

- La cooperación en los campos social y humano.

- Diálogo de culturas y civilizaciones.

- La concertación política sobre los grandes temas de la actualidad Internacional.

A partir de esta cumbre, el diálogo $5+5$ se ha ido abriendo progresivamente, intentando aprovechar su carácter informal y flexible para impulsar cooperaciones reforzadas entre los países del diálogo $5+5$, de carácter eminentemente operativo y práctico, que se pudieran plasmar en acciones concretas, en los siguientes campos: asuntos de interior, migraciones, relaciones parlamentarias, defensa, turismo y transportes.

Esta iniciativa, si bien parece tener más porvenir que los procesos euro-mediterráneos, adolece también de unos objetivos claros en la transformación de las relaciones en el Mediterráneo occidental. 


\section{Las relaciones con Argelia v Marruecos ${ }^{5}$}

En el campo bilateral la política española hacia el Mediterráneo ha mantenido al Magreb como la zona de importancia prioritaria y a Marruecos como el interlocutor fundamental en el diálogo político y la cooperación. Si durante la presidencia de José María Aznar la relación de España con Argelia y Marruecos se acabó equilibrando, en medio de fuertes desencuentros con Marruecos y que dieron lugar a la crisis de la isla de Perejil, durante los años de Rodríguez Zapatero, esta relación quedó seriamente desequilibrada, mejorando las relaciones con Marruecos a cambio de la realización de un giro notable en la posición española con respecto a la descolonización definitiva del Sahara Occidental, por más que se dijera que se respetaban los principios.

El ministro Miguel Angel Moratinos informó ante la Comisión de Asuntos Exteriores del Congreso el 19 de mayo de 2004 de las líneas generales de la política a desarrollar por su Ministerio. El ministro resaltó en primer lugar la necesidad de recomponer las relaciones con Marruecos y que, por esta razón, el presidente del Gobierno había viajado a Casablanca, inmediatamente después del debate de investidura, en su primer viaje fuera de España. Para el ministro, recuperar el diálogo, el entendimiento, la fluidez en las relaciones, la transparencia y el respeto por el otro habían sido los objetivos de este primer encuentro. Ello, según el ministro, no era un obstáculo en el desarrollo de relaciones con Argelia que se querían también privilegiar, así como con los demás países del Magreb para recuperar una política global hacia el Magreb. En este sentido iban a promover la concertación en la Unión Europea, de forma especial con Francia, para fomentar la integración regional e iban a impulsar el desarrollo de la Unión del Magreb árabe y la revitalización del proceso de integración regional. El ministro quería y se proponía hacer del Magreb un área de diálogo, de cooperación, de estabilidad, de prosperidad y de desarrollo económico y social sostenido ${ }^{6}$.

La cuestión era cómo desarrollar la capacidad de movilizar medios y actores para afrontar estos desafíos. Esto sólo podía hacerse, como reconocía el ministro, con un fuerte apoyo de la Unión Europea, que no estaba claro poder movilizar, y la experiencia del proceso de Barcelona era suficientemente concluyente. El apoyo de Francia era importante, pero no decisivo, y aquí radicó posteriormente una de las principales debilidades del plan inicial de Sarkozy de convertir la iniciativa para el Mediterráneo en una mera iniciativa regional. Además estaba sin solucionar un problema central, el conflicto no resuelto del Sahara Occidental. El ministro señaló en su comparecencia ante la Comisión de Asuntos Exteriores del Congreso que el Gobierno iba a propiciar el diálogo entre todas las partes en el conflicto del Sáhara occidental, de acuerdo con la Resolución 1495 del Consejo de Seguridad que recogía el Plan Baker II para la libre determinación del pueblo del Sáhara. El ministro reconocía que, aunque la intermediación y los buenos oficios correspondían al secretario general de Naciones Unidas y a su enviado especial, el señor Baker, el Gobierno español iba a contribuir en la búsqueda de un consenso entre las partes para buscar una solución política al conflicto, de acuerdo con la Resolución 1541, y recomponer el diálogo ${ }^{7}$.

\footnotetext{
5 Sobre la política hacia el Magreb véase de Larramendi, Miguel Hernando y Mañé Estrada, Aurelia (eds.) (2009): La política exterior española hacia el Magreb. Actores e intereses, Barcelona, Ariel.

6 "Comparecencia del Ministro de Asuntos Exteriores y Cooperación, Miguel Ángel Moratinos Cuaybé, ante la Comisión de Asuntos Exteriores del Congreso, para informar sobre las líneas generales de la política de su departamento, la política que piensa desarrollar en el Ministerio, las prioridades y políticas que piensa impulsar en relación con las materias vinculadas a esta Comisión durante la presente Legislatura”, BOCG n 24 (19 de mayo de 2004).

${ }^{7}$ Ibid.
} 
En otra intervención en el Pleno del Congreso, el ministro de Asuntos Exteriores, respondiendo a una pregunta del diputado Gustavo de Arístegui, el 19 de mayo de 2004, señaló que la voluntad de este Gobierno, sin olvidar en ningún momento el marco esencial de Naciones Unidas y el apoyo a los esfuerzos del secretario general y de su enviado especial, era la de facilitar una solución justa, duradera, consensuada, una solución política, en la que todas las partes pudieran sentirse satisfechas, que favoreciera la estabilidad del Magreb y la relación entre el Magreb y Europa. El Gobierno no quería tener una neutralidad activa y quería comprometerse en la solución.

La realidad resultó mucho más rocosa y menos transitable de lo que presentaba el ministro. La pretensión de fomentar el gran Magreb por parte española contribuyendo a solucionar este contencioso resultó un asunto demasiado arduo y excesivamente voluntarista. Esto producirá un enfriamiento de relaciones con Argelia e inducirá de nuevo medidas de retorsión por parte de Argelia. Los problemas de REPSOL con SONATRACH se han visto siempre desde una perspectiva más política que económica. En realidad, Argelia sigue siendo la asignatura pendiente española en el Magreb, yendo más allá de la energía, la cooperación policial y judicial, los flujos migratorios y el Instituto Cervantes, no siendo comparable ni la cooperación ni el tejido de relaciones creado con Marruecos con la cooperación y el tejido de relaciones existente con Argelia.

\section{La cuestión del Sahara Occidental}

Hay que recordar que España había defendido desde 1975 que el proceso de descolonización del Sahara Occidental era un proceso inconcluso que sólo se resolvería con la realización de un referéndum de autodeterminación.

Esta línea se mantendrá incluso cuando Francia en 2001 trató de persuadir a la Unión Europea para que apoyase una autonomía limitada del Sahara occidental dentro de Marruecos. España acabó arrastrando a Portugal, Irlanda, Suecia y Finlandia para mantener el apoyo a la realización de un referéndum de autodeterminación. Y Estados Unidos apoyó finalmente la fórmula de la autodeterminación.

Por otra parte, el plan Baker II tuvo también una vida corta, dada la oposición de Marruecos y James Baker acabó dimitiendo en junio de 2004, un mes después de que el ministro español hiciese público su apoyo a un plan que no se podía poner en práctica. El nuevo enviado especial personal del secretario general de la Naciones Unidas, Alvaro Soto, tuvo una duración limitada en el puesto y traía mala fama de su anterior actuación en Chipre por los retoques realizados al plan de arreglo de Chipre. En julio de 2005 tuvo lugar el nombramiento como representante especial de Peter van Walsum quien, como todos los representantes anteriores, empezó manteniendo contactos con las partes y los Estados vecinos para recabar sus opiniones sobre 1os próximos pasos que deberían darse. Al poco tiempo, de octubre de 2005 a marzo de 2006 tuvieron lugar incidentes en El-Aaiún y en algunas de las principales ciudades del Sahara occidental, produciéndose denuncias por la violación de los derechos humanos por parte de Marruecos. La situación en vez de mejorar, empeoraba.

En esta tesitura, Peter van Walsum hizo un repaso de los actores y admitió que la posición del Frente Polisario, que contaba con el apoyo general de Argelia, era que el único modo de avanzar consistía en aplicar el plan de paz para la libre determinación del pueblo del Sáhara Occidental o el plan de arreglo. Ambos habían sido aprobados o apoyados por el 
Consejo de Seguridad y contemplaban la libre determinación mediante un referéndum que incluyera la independencia entre las opciones a elegir. Cualquier otra alternativa sería inaceptable para el Frente Polisario. Mauritania había reiterado su estricta neutralidad ${ }^{8}$

En su opinión cabían dos opciones: la prolongación indefinida del estancamiento existente, en espera de una realidad política diferente, o las negociaciones directas entre las partes.

Para la mayoría de los Estados estaban en juego dos factores: el hecho de que el Sáhara Occidental no constituyera una prioridad en sus agendas políticas nacionales; y el hecho de que se concediera gran importancia a la continuidad de las buenas relaciones tanto con Marruecos como con Argelia. La combinación de estos dos factores constituía una poderosa tentación para aceptar la continuidad del estancamiento, al menos durante algunos años más. Mientras el Sáhara Occidental no adquiriese una mayor prioridad en sus agendas políticas, muchos Estados seguirían considerando que el statu quo era más tolerable que cualquiera de las posibles soluciones. Había también una tercera opción posible, que el Consejo de Seguridad impusiera una solución, obligando a Marruecos a aceptar un referéndum, con la independencia como una de las opciones. No obstante, las consultas ulteriores confirmaron que esta solución no era realista.

Así se llegó a la Resolución 1754 del Consejo de Seguridad de 30 de abril de 2007 que exhortó a las partes a entablar negociaciones sin condiciones previas, teniendo presentes los acontecimientos de los últimos meses, con miras a lograr una solución política justa, duradera y mutuamente aceptable que condujera a la libre determinación del pueblo del Sáhara Occidental y el 18 de junio tuvo lugar la primera reunión del proceso de negociación en la finca de Greentree en Manhasset, Nueva York. Una segunda reunión se celebró en agosto sin conseguirse avances.

Estaba ya claro, que frente a lo que mantenía Peter Van Walsum, (“Argelia desempeña un papel preeminente y dominante") Argelia no quería aparecer como parte en la negociación y era también meridianamente claro que España no había conseguido modificar el contexto y que no tenía capacidad de influencia suficiente. De esta forma, se mantuvo la cuestión del derecho de autodeterminación, dado que la anexión no encontró apoyos suficientes.

En enero de 2008 se produjo la tercera reunión y la cuarta en marzo. El Frente Polisario no dudó en plantear la independencia. En este contexto, Peter Van Walsun manifestó en abril en el Consejo de Seguridad que el Frente Polisario debía adoptar una actitud realista y renunciar a la independencia. De inmediato el Polisario pidió la dimisión de Van Walstun. Y, como era de esperar, el ministro Miguel Ángel Moratinos apoyó a Van Walstun. El resultado fue la ruptura del proceso. La política española quedaba en evidencia frente a lo que el ministro de Asuntos Exteriores en su día defendió, señalando que "España, por su capacidad de influencia en la región, puede aportar un plus para encontrar una salida".

Esta pretensión acabó por ser devaluada aún más por el empuje de los grupos prosaharauis españoles que han dejado un estrechísimo margen de maniobra al gobierno, obligando al partido socialista a adoptar una posición de mayor compromiso con el Frente Polisario. El caso de Aminattou Haidar así como las continuas denuncias de violaciones de

\footnotetext{
8 También había mantenido consultas con las autoridades del Reino Unido, España, Francia, y los Estados Unidos de América en Londres, Madrid, París, y Washington, D.C., así como con Alpha Oumar Konare, Presidente de la Comisión de la Unión Africana, en Addis Abeba y con altos cargos de la Unión Europea en Bruselas
} 
los derechos humanos por Marruecos y los enfrentamientos entre los habitantes del Sahara Occidental y las fuerzas policiales de Marruecos han obligado al gobierno a tomar una mayor distancia en este asunto.

En términos generales podemos decir que España consiguió sin ningún género de dudas unas relaciones más fluidas con Marruecos, favoreciendo también unas especiales relaciones de Marruecos con la Unión Europea, privilegiando la cooperación económica gubernamental y no gubernamental, la apertura de mercados y obteniendo, como cuestiones fundamentales, la renovación del acuerdo de pesca, una colaboración en el tema de lucha contra la emigración ilegal, con sus altos y bajos, y una colaboración en el tema terrorista que los atentados del 11 de marzo de 2004, de impronta claramente marroquí, habían puesto sobre la mesa como asunto prioritario. Pero la política global quedaba desequilibrada con respecto a Argelia, lo que obliga a replantear la, así denominada, "visión global”.

\section{Oriente Próximo}

Con respecto a Oriente Próximo el nuevo Gobierno socialista, tal como había prometido, decidió poner fin a la participación militar española en Irak, pero lo hizo de forma brusca, e incluso alentando a terceros Estados que tenían tropas en Irak a que siguieran la decisión de España de retirar sus efectivos militares de este país porque así se abriría "una expectativa más favorable". El presidente José Luis Rodríguez Zapatero hizo esta consideración en la conferencia de prensa que cerró la Reunión de Alto Nivel hispano-tunecina el 13 de septiembre de 2004. Esto produjo la indignación de los Estados Unidos. El presidente Bush ya ni siquiera devolvió las llamadas al presidente español. Sobre el terreno, la invasión de Irak por la coalición no favoreció la solución de los conflictos árabe-israelí y palestino israelí, antes bien la empeoró.

España, con el nuevo gobierno socialista, apoyó la permanencia de un Irak unido, estable, seguro, democrático y próspero, subrayando que era necesario que el pueblo iraquí recuperase cuanto antes el control sobre su propio país.

Sobre el proceso de paz de Oriente Próximo, el gobierno se propuso avanzar mediante la colaboración entre los Estados del Mediterráneo y el mundo árabe, restableciendo la confianza entre los pueblos, favoreciendo una negociación entre las partes y respetando las resoluciones de Naciones Unidas, los principios de la Conferencia de Paz de Madrid y los acuerdos posteriores aceptados por las partes. De este modo, decía el ministro de Asuntos Exteriores en su primera comparecencia ante la Comisión de Asuntos Exteriores del Congreso, "podrá alcanzarse un acuerdo de paz definitivo basado en la existencia de dos Estados soberanos, viables y seguros, dentro de fronteras seguras y reconocidas, tal como establece la Hoja de Ruta, y el Gobierno seguirá apoyando los esfuerzos del cuarteto".

En realidad las posibilidades de influencia española en estos conflictos han sido siempre limitadas. Por otra parte, ni la Unión Europea, que ha estado siempre divida, y que, como dijimos, se ha dedicado más que a hacer política a la gesticulación en este asunto, ni los Estados Unidos durante la Administraciones del presidente Bush y del presidente Obama han conseguido reorientar la posición israelí, siempre manteniendo enormes reservas sobre el resultado de las negociaciones, mucho más tras la elección del presidente Netanyahu quien no cree en el proceso de paz. 
Para España una paz global y duradera en la región también tenía que incluir una solución para los problemas de Siria y Líbano, de acuerdo con las resoluciones de Naciones Unidas.

En términos más generales, la visión del Ministerio de Asuntos Exteriores, tras los procesos electorales llevados a cabo en 2005 y 2006 en la zona, se concretó en que existía una interdependencia entre los conflictos en la región, que el conflicto central, a pesar de las consecuencias de la guerra de Irak, era el conflicto árabe-israelí y así lo dijo el presidente José Luis Rodríguez Zapatero ante la Asamblea General de la ONU en septiembre de 2004: "no habrá seguridad ni estabilidad en el mundo mientras sangre el conflicto de Oriente Próximo, que es el tumor primario de múltiples focos de inestabilidad"; y para la resolución de estos conflictos había que pasar de la gestión de crisis al fomento y creación de espacios de cooperación e integración.

En este esquema se ha movido la política española. España se integró en la FINUL, desplegándose un contingente español de 1.100 efectivos tras el cese de hostilidades entre Israel y el Líbano, el 15 de septiembre de 2006. La cuestión es la efectividad de este tipo de despliegues, cuya lógica es ayudar a las partes a que encuentren una solución, no una permanencia en la zona sin ningún avance en la solución del conflicto entre las partes. En cualquier caso, dado el interés de España por el Mediterráneo, no podía quedar fuera de una iniciativa que tenía una impronta europea.

España y la Unión Europea apoyaron asimismo al presidente Abas frente a Hamas como representante legítimo de los palestinos. Además, a pesar de los problemas que Irán ha creado en la zona, se intentó desde el primer momento mantener abiertas las vías de diálogo con el gobierno de Irán, tal como había pretendido el gobierno del presidente Aznar, en un contexto de política interna iraní diferente.

Desde el punto de vista de las relaciones con Israel la voluntad de acercamiento manifestada por el ministro Moratinos ha venido lastrada por el peso de la opinión pública española más favorable a la causa palestina.

Digno también de mención ha sido el desarrollo de las relaciones con Turquía, favoreciendo su integración en la Unión Europea, desarrollando las relaciones económicas y lanzando ambos presidentes la iniciativa Alianza de Civilizaciones, una iniciativa interesante, pero inicialmente muy mal planteada teóricamente ${ }^{9}$ dando lugar a un amplio desinterés, a pesar del dinero invertido en su promoción, su obligatoriedad de estudio en currículos oficiales; iniciativa a la que el propio presidente del gobierno, inasequible al desaliento, se ha agarrado hasta el final.

En otro orden, el nuevo gobierno socialista creó en Madrid la Casa Árabe y, posteriormente, la Casa Sefarad, en un intento de reforzar el conocimiento del mundo árabe y el mundo judío en España.

\footnotetext{
${ }^{9}$ Véase a este respecto Marquina, Antonio: "Nota Editorial", UNISCI Discussion Papers, no 14 (mayo 2007), en http://www.ucm.es/info/unisci/revistas/UNISCI14Nota.pdf.
} 


\section{El contencioso de Gibraltar y las ciudades españolas de Ceuta y Melilla}

De forma simultánea veremos la realización de un giro adanista español en el contencioso de España sobre Gibraltar y la creación del Foro de diálogo sobre Gibraltar. Este es un tema especialmente vidrioso, dados los bandazos que el gobierno socialista desde los años noventa ha realizado en este tema sin ningún escrúpulo, incluso recurriendo a la desinformación ${ }^{10}$ y la incuria con que se ha llevado este tema en los Ministerios de Asuntos Exteriores y también de Defensa, a pesar de mantenerse que el estrecho de Gibraltar es una zona de interés estratégico primordial de España.

España durante los gobiernos del presidente José María Aznar impulsó el proceso de descolonización incidiendo en el tema de la soberanía. Tras el fracaso de las negociaciones llevadas a cabo por los gobiernos español y británico entre 2001 y 2002 en un intento de llegar a un acuerdo de cosoberanía, siendo ministro de Asuntos Exteriores, Josep Piqué, la cuestión gibraltareña quedó bloqueada. El acuerdo no se llegó firmar, pues las autoridades gibraltareñas convocaron un referéndum donde, como era previsible, fue ampliamente rechazado.

Ante dicho bloqueo, aceptado por el Foreign Office, el nuevo ministro de Asuntos Exteriores y Cooperación, Miguel Ángel Moratinos manifestó inicialmente ante la Comisión de Asuntos Exteriores del Congreso que el Gobierno tenía el firme propósito de recuperar e impulsar el diálogo y la negociación en el contexto del proceso de Bruselas, con el espíritu que alentó el ministro hasta julio de 2002, añadiendo "Con ese objetivo mañana mismo voy a reunirme en Londres con el secretario del Foreign Office, Jack Straw, con el que voy a abordar la cuestión de Gibraltar en sus dos aspectos más relevantes: la soberanía y la cooperación. Estoy convencido de que mediante el diálogo podemos avanzar en el proceso negociador y conseguir beneficios para todas las partes implicadas en la cuestión de Gibraltar, incluyendo por supuesto a la región circunvecina, es decir, al Campo de Gibraltar"11.

Pero al poco tiempo, en octubre de 2004, hace su aparición el Foro Tripartito de Diálogo sobre Gibraltar ${ }^{12}$, creándose formalmente en diciembre de 2004, desechándose por parte española la condición de que debía haber progresos en soberanía para que hubiera a su vez progresos en cooperación. En el Foro quedaban representados España, el Reino Unido y Gibraltar y el diálogo era a tres bandas, con agenda abierta, y donde Gibraltar tenía voz propia y las decisiones en asuntos de cooperación habían de ser acordadas por los tres participantes. El 18 de septiembre de 2006 se firmaron los acuerdos de Córdoba que incluían la utilización por España de la pista de aterrizaje de $\operatorname{Gibraltar}^{13}$, el tránsito por la verja construida por el

\footnotetext{
${ }^{10}$ Véase a este respecto Antonio Marquina: La otra batalla de Gibraltar, UNISCI Discussion Papers, $\mathrm{n}^{\circ} 4$ (enero de 2004), en http://www.ucm.es/info/unisci/revistas/Gibraltar.pdf.

11 “Comparecencia del Ministro de Asuntos Exteriores y Cooperación, Miguel Ángel Moratinos Cuaybé, ante la Comisión de Asuntos Exteriores del Congreso, para informar sobre las líneas generales de la política de su departamento, la política que piensa desarrollar en el Ministerio, las prioridades y políticas que piensa impulsar en relación con las materias vinculadas a esta Comisión durante la presente Legislatura”, BOCG n 24 (19 de mayo de 2004).

12 Véase Inmaculada García, Alejandro del Valle (2010): Gibraltar y el Foro Tripartito de Diálogo, Madrid, Dykinson, S.L.-Libros.

${ }^{13}$ Sobre la importancia de la pista de aterrizaje y los aspectos estratégicos de Gibraltar véase Romero, Luis: "Lo estratégico en la cuestión de Gibraltar", UNISCI Discussion Papers, n ${ }^{\circ} 12$ (octubre 2006), en http://www.ucm.es/info/unisci/revistas/UNISCIRomero12.pdf.; Marquina, Antonio: "La pista de aterrizaje de Gibraltar y la base militar", UNISCI Discussion Papers, no 19 (enero 2009), en http://www.ucm.es/info/unisci/revistas/UNISCI\%20DP\%2019\%20-\%20MARQUINA\%20GIBRALTAR.pdf.
} 
Reino Unido, las pensiones a los españoles que trabajaron en Gibraltar y las telecomunicaciones.

Posteriomente, el 21 de julio de 2009 tuvo lugar la primera visita oficial a Gibraltar de un miembro del Gobierno español, Miguel Ángel Moratinos, asunto que levantó una importante polémica y dividió a las fuerzas políticas españolas, dado que el tema de la soberanía seguía aparcado y significaba dar un espaldarazo a las autoridades de la colonia, mientras se seguía produciendo la expansión de Gibraltar en aguas españolas, expansión convertida en un mal endémico, con el apoyo español al utilizarse tierras y materiales traídos desde España en proyectos faraónicos, que en 2006 se evaluaba su coste en más de mil millones de libras, sin que el gobierno español les pusiera coto, la política británica de legitimación de hechos consumados, con el otorgamiento de una nueva Constitución, las afirmaciones del ministro principal sobre no reconocimiento del Comité de Descolonización de Naciones Unidas, la cuestión de los submarinos atómicos que seguían recalando en Gibraltar, los vertidos y la polución de la bahía por accidentes y las actividades de bunkering en Gibraltar, y un largo etcétera de incidentes que fueron creciendo en los meses posteriores entre las patrulleras de la Guardia Civil y los buques de la Marina británica en aguas situadas en las tres millas cercanas a la costa de Gibraltar, aguas nunca cedidas por España.

El colmo del disparate tuvo lugar cuando el ministro principal de Gibraltar retó al ministro de Asuntos Exteriores español a llevar esta disputa a la Corte Internacional de Justicia.

El Foro permitió que Peter Caruana se colocara en una situación de igualdad con los otros dos ministros sin ofrecer nada a cambio. De los seis campos de cooperación acordados en la visita del ministro español a Gibraltar sólo se había avanzado en tres: fiscal; educativo y cultural; y visados, mientras que los capítulos de cooperación policial, judicial y aduanera; medio ambiente; y comunicaciones y seguridad marítima estaban atascados, siendo los de más interés para España.

El resultado del adanismo español ha sido un desastre sin paliativos. El planteamiento alternativo que se trató desarrollar confundía los deseos con la realidad.

En el caso de Ceuta y Melilla las posiciones españolas se han mantenido formalmente. En este asunto las posiciones tradicionales del partido Popular y del Partido Socialista han sido dispares, no cabe decir otra $\cos ^{14}$.

En los últimos años, aparentemente y teóricamente la posición de estas ciudades españolas se han visto fortalecidas con las visitas de Rodríguez Zapatero a ambas ciudades en 2006 y del Rey en 2007, pero el proceso de venta de propiedades a ciudadanos de origen marroquí se ha incrementado. El resultado acabará siendo una cesión silenciosa.

\section{Las relaciones económicas con los Estados del sur del Mediterráneo}

En el campo económico la presencia española en el mundo árabe, saliendo del Magreb sigue siendo una asignatura pendiente- yendo más allá de la venta de armamento- y en el campo de

\footnotetext{
${ }^{14}$ Véase a este respecto García Flórez, Dionisio (1999): Ceuta y Melilla, Cuestión de Estado, Ciudad Autónoma de Melilla. García, Dionisio (1998): Ceuta y Melilla en la política española. Perspectivas futuras, UNISCI Paper, $n^{\circ} 13$, Madrid, UNISCI.
} 
la cooperación sería oportuna una mejor distribución de recursos entre países. Una excepción en el Mediterráneo lo constituye Turquía, pero Turquía no es un Estado árabe.

Hay que hacer notar que la crisis económica lo que está permitiendo es la presencia económica de grupos inversores árabes, asunto interesante que hubiera exigido una mejor planificación española.

Las cifras de inversiones y exportaciones de la última década son suficientemente concluyentes. En Argelia se ha pasado de exportar por un valor de 548 millones de euros en el año 2000 a 2.037 en el año 2010, mientras que las importaciones han pasado de los 3.094 millones a 4.551, teniendo un pico en 2008 de 6.431 millones, con lo que el gap comercial se ido recortando. En el caso de Marruecos, por el contrario, se ha pasado de unas exportaciones de 1.371 millones de euros en el año 2000 a 3.449 en 2010 y las importaciones han crecido también muy sustancialmente pasando de los 967 millones a los 2.775. En el caso de Libia las importaciones han subido de 2.030 millones de euros en el año 2000 a 3.352 en 2010, pero las exportaciones han crecido muy poco, pasando de 119 millones a 259 en los mismos años, siendo el gap comercial muy grande. Con Túnez las cifras comerciales son favorables a España y en el caso de Egipto se ha pasado de un superávit en la balanza comercial en el año 2000 a un desequilibrio muy notable a partir del año 2005, alcanzando las exportaciones españolas la cifra de 912 millones de euros frente a las importaciones de 1.345 millones en 2010. La balanza comercial es favorable a España en el caso de Líbano y Jordania y bastante equilibrada en el caso de Siria, si bien los montantes de exportaciones e importaciones en el Mediterráneo oriental son algo mediocres con la excepción de Israel. Más allá, con los Estados del Golfo e Irán la balanza comercial está profundamente desequilibrada en la mayoría de los casos. España tiene esta asignatura pendiente desde hace más de una década.

En cuanto a inversiones españolas, los flujos de inversión bruta española en la década del 2000 son significativos, siendo Marruecos (3.247 millones de euros) el Estado más beneficiado, a gran distancia del segundo que es Egipto (694 millones), el tercero es Túnez (265 millones), el cuarto Argelia (258 millones) y ya el quinto a gran distancia es Arabia Saudita (127 millones). En Libia, Líbano, Siria e Israel la inversión es reducida y casi ridícula.

\section{Conclusión}

Como indicamos al inicio de este artículo esta última década ha supuesto cambios de importancia en la política española hacia el Mediterráneo. Lamentablemente hay que decir que España ha perdido fuerza en sus políticas mediterráneas y de forma especial en el Magreb y el estrecho de Gibraltar, exigiéndose nuevos diseños, planteamientos más ajustados a las posibilidades españolas, acercamientos a los Estados emergentes con presencia en el Mediterráneo y más refinadas alianzas a nivel europeo y con los Estados Unidos. La primavera árabe abre incógnitas y exigirá unos planteamientos distintos en muchos casos para adaptarse al nuevo contexto geopolítico, socio-económico, religioso y cultural mediterráneo, un Mediterráneo ampliado y diferente al de las últimas décadas. 